\title{
Relating hepatocellular carcinoma tumor samples and cell lines using gene expression data in translational research
}

\author{
Bin Chen, Marina Sirota, Hua Fan-Minogue, Dexter Hadley, Atul J Butte \\ From The 4th Translational Bioinformatics Conference and the 8th International Conference on Systems \\ Biology (TBC/ISB 2014) \\ Qingdao, China. 24-27 October 2014
}

\begin{abstract}
Cancer cell lines are used extensively to study cancer biology and to test hypotheses in translational research. The relevance of cell lines is dependent on how closely they resemble the tumors being studied. Relating tumors and cell lines, and recognizing their similarities and differences are thus very important for translational research. Rapid advances in genomics have led to the generation of large volumes of genomic and transcriptomic data for a diverse set of primary cancer samples, normal tissue samples and cancer cell lines. Hepatocellular Carcinoma (HCC) is one of the most common tumors worldwide, with high occurrence in Asia and sub-Saharan regions. The current effective treatments of HCC remain limited. In this work, we compared the gene expression measurements of 200 HCC tumor samples from The Cancer Genome Atlas and over 1000 cancer cell lines including 25 HCC cancer cell lines from Cancer Cell Line Encyclopedia. We showed that the HCC tumor samples correlate closely with HCC cell lines in comparison to cell lines derived from other tumor types. We further demonstrated that the most commonly used HCC cell lines resemble HCC tumors, while we identified nearly half of the cell lines that do not resemble primary tumors. Interestingly, a substantial number of genes that are critical for disease development or drug response are either expressed at low levels or absent among highly correlated cell lines; additional attention should be paid to these genes in translational research. Our study will be used to guide the selection of HCC cell lines and pinpoint the specific genes that are differentially expressed in either tumors or cell lines.
\end{abstract}

\section{Introduction}

Cancer cell lines are widely used to study cancer biology and to test hypotheses in translational research [1,2]. Numerous cancer cell lines are derived from tumors, and are used for various purposes such as understanding disease progression, developing diagnostics, and screening anti-tumor drugs. However, the relevance of a cell line is dependent on how closely it resembles the tumors being studied, while the in vitro cell culture environment differs from that of in vivo tumor tissue. Moreover, not all cancer cell lines have equal value or relevance as tumor models [3]. Therefore, relating tumors and cell lines, and

\footnotetext{
* Correspondence: abutte@stanford.edu

Division of Systems Medicine, Department of Pediatrics, Stanford University School of Medicine, 1265 Welch Road MSOB X163, Stanford, CA, 94305-5415,
} USA

recognizing their similarities and differences is of critical importance in translational research and understanding cancer biology.

It has been shown that genetic and epigenetic changes including gene mutations, deletions, amplifications, translocations and methylation status found in lung tumors are retained in lung cancer cell lines [4]. Prolonged cell culture is more likely to cause the secondary genomic changes such as copy number variation and gene expression [3]. Several previous studies have compared the genomic and transcriptomic differences between tumors and cell lines, but their sample size has been very limited [5-8]. Integrating disease tissue gene expression and drug gene expression profiled in cancer cell lines for therapeutic discovery has been extensively applied by our lab and others [9-13]. Rapid advances in 
the field of genomics have led to the generation of a high volume of molecular data across various tumors and cell lines. Of particular note, The Cancer Genome Atlas (TCGA) Research Network has characterized the genomic and gene expression profiles of over 10,000 human tissue samples cross 32 tumor types [14]. The Cancer Cell Line Encyclopedia (CCLE) provides the genomic and gene expression profiles of over 1000 cell lines [15]. Domcke et al. recently evaluated ovarian cell lines as tumor models by comparing their genomic profiles published in CCLE and TCGA, and they found that some rarely used cell lines might be more appropriate to study ovarian cancers [16]. To our knowledge, here we present the first comprehensive comparison of tumor and cell line samples using gene expression data specifically for liver cancer.

Liver cancer is the sixth most frequent cancer globally with higher occurrences in Asia and sub-Saharan regions and is the second leading cause of global cancer deaths. The incidence and mortality of liver cancer have increased in the United States and Europe in the past decade. It was projected to become the third leading cause of cancer-related death by 2030 in the USA [17]. While HCC is the most common type of liver cancer, the current effective treatments of HCC remain limited. HCC tumors are insensitive to conventional chemotherapy, although limited benefits have been shown from the targeted drug sorafenib on only a small subset of tumors [18]. Meanwhile, a large number of liver cell lines have been developed and used to screen compounds, and many drugs are showing promising in vitro models. However, that these best drug candidates fail to be effective clinically [19] indicates the necessity to examine the correlation between HCC tumors and cancer cell lines and elucidate their similarities and differences for more effective translation of bench-side discovery into clinical utility.

In this work, we compared $200 \mathrm{HCC}$ tumor samples from TCGA to over 1000 cancer cell lines from CCLE using their gene expression profiles, and we found that most HCC cell lines are significantly correlated to the primary tumors in comparison to other cancer cell lines. However, from the set of $25 \mathrm{HCC}$ cell lines, nearly half are not significantly correlated to the tumors. We also found that a small subset of the tumors is not significantly correlated to HCC cell lines. We further identified the differentially expressed genes between the tumor samples and cell lines, and we found that a substantial number of differentially expressed genes are located in the extra cellular space. The genes that are overexpressed in the tumor samples are related to immune response, drug metabolism and $\mathrm{ABC}$ transporter. This work helps guide us in optimal selection of cell lines and highlights the differentially expressed genes that play an essential role in translating from in vitro to in vivo and clinical research.

\section{Results \\ Correlation of gene expression between HCC tumor samples and cancer cell lines}

We compared 200 HCC tumors profiled by RNASeq from TCGA with 1019 cancer cell lines profiled by microarray from CCLE using the top 5000 varying genes (see Methods). There are 28 cancer cell lines derived from liver tissues and 25 of them are related to HCC. Fourteen HCC related cell lines are significantly correlated to the tumors, and 7 non-liver cancer cell lines are correlated ( $P$ value $<0.05$, Figure 1a). Of particular note, pancreatic cell line TCC-PAN2 (median correlation coefficient of $0.49, \mathrm{P}$ value $=8.8 \mathrm{E}-3)$ and stomach cell line FU97 (median correlation coefficient of 0.50, P value $=5.5 \mathrm{E}-3$ ) are even more correlated with the $\mathrm{HCC}$ tumors than some of the HCC cell lines. This suggests that quite a few cell lines originated from another site share similar gene expression profiles with HCC tumors, and they may be used to inform the study of HCC as well.

Among 24 cell line groupings differentiated by their tissue of origin, we found liver cancer cell lines are most correlated to the HCC tumors (median correlation coefficient of 0.44 , Figure 1b). The overall correlation is significantly higher than the second and third most correlated cell types, which are the kidney and biliary tract cancer cell lines (median correlation coefficient of 0.41 , $P$ value $<1 \mathrm{E}-16$; 0.40, $\mathrm{P}$ value $<1 \mathrm{E}-16$, respectively). The three least correlated cell line types are autonomic ganglia (median correlation coefficient of 0.22 ), hematopoietic and lymphoid tissue (median correlation coefficient of 0.31 ) and small intestine (median correlation coefficient of 0.32). According to TCGA tissue sample requirements, all tumors were taken at the initial site of cancer. Thus we conclude that HCC cell lines retain the characteristics of the liver origins.

\section{Correlation of gene expression between HCC tumors and $\mathrm{HCC}$ cell lines}

However, when looking closely at the distribution of the correlations between HCC tumors and HCC cell lines, we notice that the variation is rather high, with value ranging from 0.19 to 0.66 , and standard deviation 0.07 (Figure 1b).

Moreover, we observe that there are two groups of cell lines and one group is more correlated with the tumor samples than the other on the hierarchical clustering of the individual cell lines (Figure 2a). The four most commonly used cell lines (i.e., HepG2, HuH 7, Hep3B and PLC/PRF/5) [19] have significantly high correlations with the tumor samples ( $\mathrm{P}$ value $<0.05$ ). 


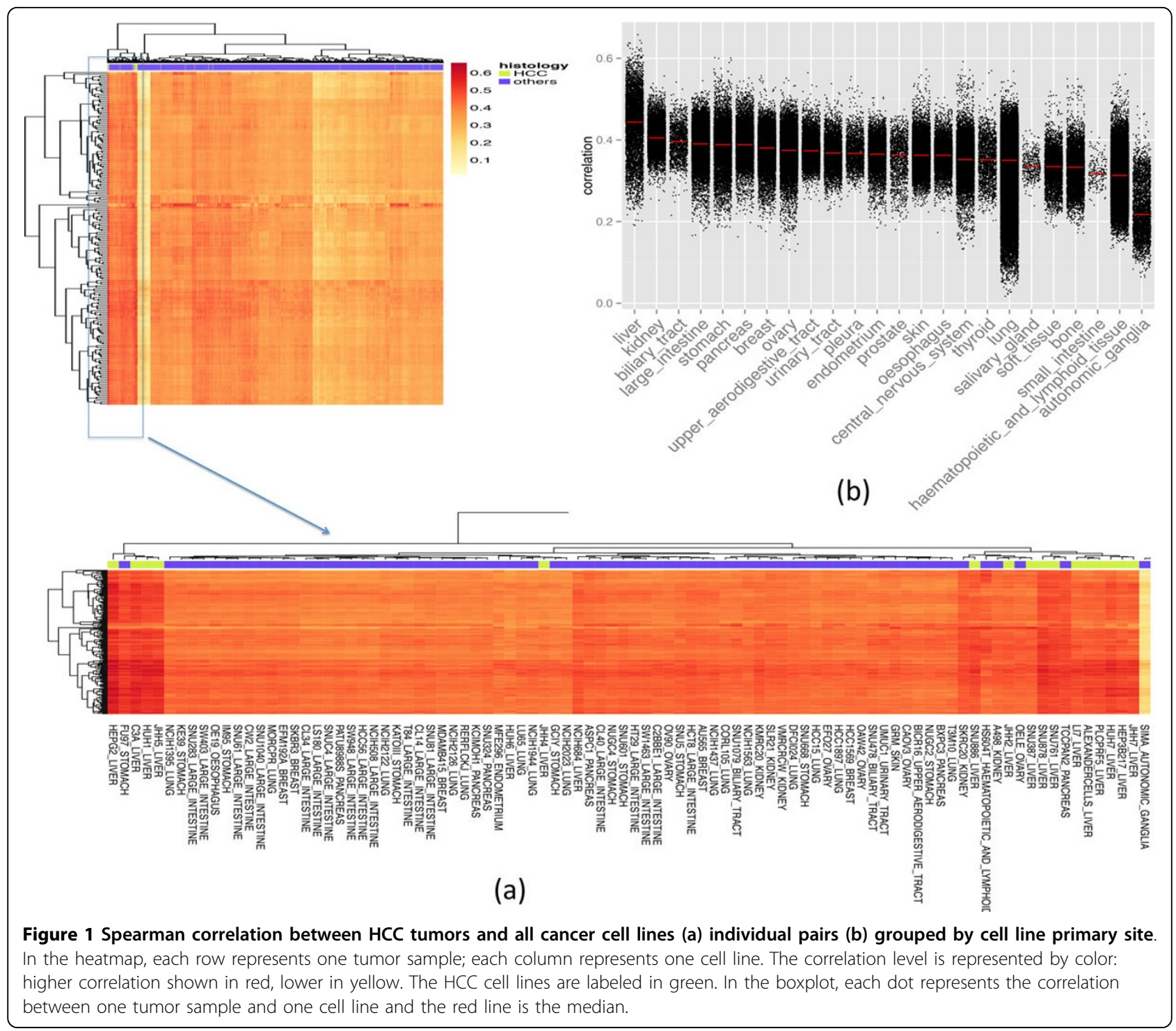

Interestingly, HepG2, one of the most widely used cell line, has the highest correlation (median correlation coefficient of $0.55, \mathrm{P}$ value $=3.4 \mathrm{E}-4)$. However, 11 out of 25 cell lines are not significantly correlated to the tumor samples by comparing to other cell lines ( $\mathrm{P}$ value $>0.05$ ).

Table 1 lists all HCC cell lines and the medians of their correlation coefficients across the $200 \mathrm{HCC}$ tumors, ranked by their correlation coefficient value. Although we could not find any factors that could be associated with the correlations due to the small sample size as well as incomplete information about some cell lines, several observations are noteworthy. The cell lines sharing high SNP identity (e.g., HepG2 \& C3A, PLC/ $\mathrm{PRF} / 5$ \& Alexander cells, and HLF \& HLE) tend to have similar correlations. We find that for the SNU series, all the most recently established cell lines (i.e., SNU-878,
SNU-761, and SNU-886) are highly correlated but none of the older ones are significantly correlated. The publication of the newer established cell lines did not report the explicit difference between the two versions of cell lines [20]. The cells that are not well differentiated in HLF and HLE may account for their low correlations with the tumors.

Additionally, we find that the majority of the tumor samples are individually significantly correlated to the HCC cell lines (a few samples are exampled in Figure 3a). We identify 8 tumor samples as outliers (Figure 3b), after using tissue samples randomly selected from the Expression Project for Oncology [21] to compute the false discovery rate (FDR $\leq 0.05$, see Methods). Interestingly, these 8 tumors are similar with each other and are different from the majority of tumors according to the principal component analysis of their gene expression profiles 


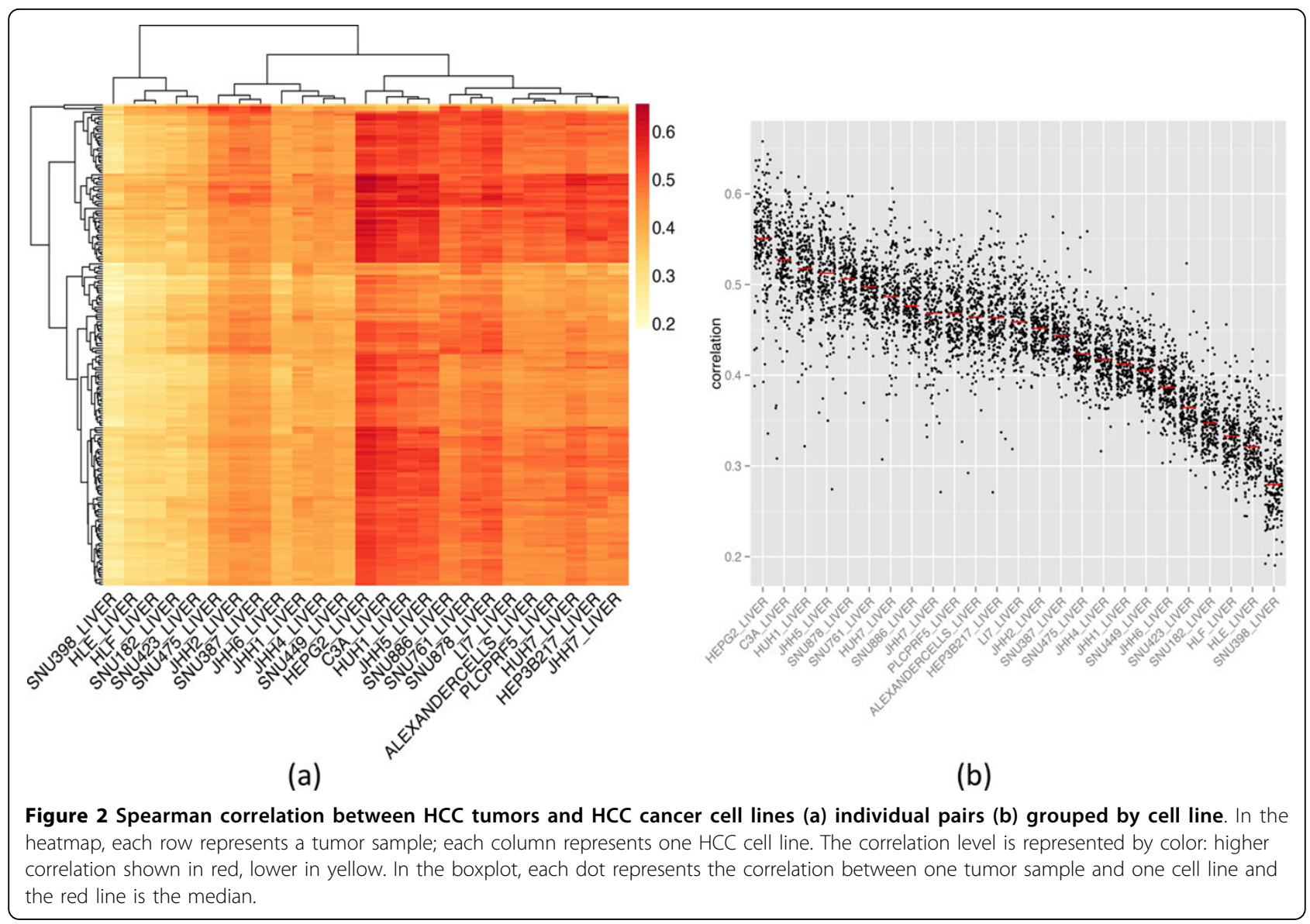

(Figure 3c). This suggests that the current cell lines may be incapable of modeling this subset of tumors based on gene expression analysis. In addition, based on the analysis of clinical features of the outlier samples (Table 2) we are not able to identify any clinical features that are significantly associated with this subset of tumors due to the small sample size.

\section{Difference of gene expression between HCC tumors and HCC cell lines}

Most HCC tumors and cell lines share remarkably similar gene expression profiles. However, since the environment of cells growing in vitro is different from that in a heterogeneous tissue, the prolonged process of cell culture may induce substantial changes of the expression of some genes. Furthermore drug response in vitro is different from that in vivo or in the clinic. For example, HCC tumors are often insensitive to some chemotherapy drugs while HCC cell lines are sensitive to those drugs [22]. Therefore, we hypothesize that differentially expressed genes between tumors and cell lines may play important roles in disease development or drug response.

In this regard, we first rank the commonly measured genes between HCC tumor samples and HCC cell line samples based on their expression values in individual samples. For each gene, we computed the difference of the ranks between tumor samples and cell lines to identify differentially expressed genes. Among 17,386 commonly measured genes, 385 are over expressed in tumors compared to cell lines and 279 are over expressed in cell lines compared to tumors ( $\mathrm{P}$ values < 0.01 , $\log 2$ fold change $>2$, data in the additional file 1 : Table S1-2). We then ask if these genes are relevant to disease development. We reason that the differentially expressed genes between tumors and non-tumors are related to the disease development. We find 1003 genes being significantly differentially expressed between the tumor samples and non-tumor samples from TCGA. A significant number of over expressed genes in tumors compared to cell lines are dysregulated in tumors compared to non-tumors (30 common genes, $\mathrm{P}$ value = 0.008 , hypergeometric test, data in the additional file 1 : Table S3-5). This suggests that the over expressed genes in tumors compared to cell lines are indeed important for the disease development.

We further perform GO and KEGG pathway enrichment analysis of the differentially expressed genes between tumor samples and cell lines using DAVID [23]. 
Table 1. HCC cell lines and their correlations with tumors

\begin{tabular}{|c|c|c|c|c|c|}
\hline Cell Line & $\begin{array}{c}\text { Correlation } \\
(P \text { value })\end{array}$ & Sex & Ethnicity/Race & Age & Known cancer etiology or other characteristics \\
\hline HepG2 & $0.55(3.4 \mathrm{E}-4)$ & M & Caucasian/NA & 15 & No evidence of a Hepatitis B virus genome \\
\hline$\overline{C 3 A}$ & $0.53(1.1 \mathrm{E}-3)$ & M & Caucasian/NA & 15 & No evidence of a Hepatitis B virus genome; derivative of HepG2 \\
\hline $\mathrm{huH}-1$ & $0.52(1.9 \mathrm{E}-3)$ & M & Asian/Japanese & 53 & HBs-antigen carrier \\
\hline $\mathrm{JHH}-5$ & $0.51(3.3 \mathrm{E}-3)$ & M & Asian/Japanese & 50 & $\begin{array}{l}\text { Small liver cancer observed in the chronic hepatitis; integration of the HBV-DNA was } \\
\text { not observed }\end{array}$ \\
\hline SNU-878 & $0.51(3.3 \mathrm{E}-3)$ & $\mathrm{F}$ & Asian/Korean & 54 & One of newer cell lines in the SNU series \\
\hline SNU-761 & $0.5(5.5 \mathrm{E}-3)$ & M & Asian/Korean & 49 & One of newer cell lines in the SNU series \\
\hline $\mathrm{HuH}-7$ & $0.49(8.8 \mathrm{E}-3)$ & M & Asian/Japanese & 57 & Well differentiated hepatocellular carcinoma \\
\hline SNU-886 & $0.48(1.4 \mathrm{E}-2)$ & M & Asian/Korean & 57 & One of newer cell lines in the SNU series \\
\hline $\mathrm{JHH}-7$ & $0.47(2.1 \mathrm{E}-2)$ & M & Asian/Japanese & 53 & HBs-Ag positive hepatocellular carcinoma with liver cirrhosis \\
\hline $\mathrm{PLC/PRF/5}$ & $0.47(2.1 \mathrm{E}-2)$ & M & NA & 24 & Malignant liver cancer with HBsAg positive \\
\hline Alexander cells & $0.46(3.1 \mathrm{E}-2)$ & M & NA & 24 & Malignant liver cancer with $\mathrm{HBsAg}$ positive \\
\hline Hep 3B2.1-7 & $0.46(3.1 \mathrm{E}-2)$ & M & Black/NA & 8 & Hepatitis B virus DNA was detected \\
\hline $\mathrm{Li}-7$ & $0.46(3.1 \mathrm{E}-2)$ & NA & NA & NA & NA \\
\hline$\overline{\mathrm{JHH}-2}$ & $0.45(4.5 \mathrm{E}-2)$ & M & Asian/Japanese & 57 & Integration of the HBV-DNA was not observed \\
\hline SNU-387 & $0.44(0.06)$ & $\mathrm{F}$ & Asian/Korean & 41 & $\begin{array}{l}\text { Patient treated by transcatheter arterial embolization with lipoidol plus a combination } \\
\text { of doxorubicin and mitomycin-C }\end{array}$ \\
\hline SNU-475 & $0.42(0.12)$ & M & Asian/Korean & 43 & Taken from a patient prior to cytotoxic therapy \\
\hline $\mathrm{JHH}-4$ & $0.42(0.12)$ & M & Asian/Japanese & 51 & HBs antigen-negative; HCV was not detected \\
\hline$\overline{\mathrm{JHH}-1}$ & $0.41(0.16)$ & M & Asian/Japanese & 50 & Complication of hepatic cirrhosis and hepatocellular carcinoma \\
\hline SNU-449 & $0.41(0.16)$ & M & Asian/Korean & 52 & Hepatitis B virus DNA was detected; taken from a patient prior to cytotoxic therapy \\
\hline $\mathrm{JHH}-6$ & $0.39(0.25)$ & $\mathrm{F}$ & Asian/Japanese & 57 & HBV-DNA was not integrated; undifferentiated hepatocellular carcinoma \\
\hline SNU-423 & $0.36(0.44)$ & M & Asian/Korean & 40 & $\begin{array}{l}\text { Hepatitis B virus DNA was detected; treated by transcatheter arterial embolization with } \\
\text { lipoidol plus doxorubicin }\end{array}$ \\
\hline SNU-182 & $0.35(0.51)$ & M & Asian/Korean & 24 & Taken from a patient prior to cytotoxic therapy \\
\hline HLF & $0.33(0.64)$ & M & NA & 68 & Hepatoma, non-differentiated \\
\hline$\overline{H L E}$ & $0.32(0.70)$ & $M$ & NA & 68 & Hepatoma, non-differentiated \\
\hline SNU-398 & $0.28(0.89)$ & M & Asian/Korean & 42 & $\begin{array}{l}\text { Hepatitis B virus (HBV) DNA was detected; treated by transcatheter arterial } \\
\text { embolization with lipoidol plus a combination of doxorubicin and mitomycin-C }\end{array}$ \\
\hline
\end{tabular}

Median of correlation coefficients is used.

For those genes over expressed in tumors compared to cell lines, the top three enriched cellular components are extracellular region, extracellular space and extracellular region part, the top three enriched biological processes are immune response, response to wounding and defense response, and the top three enriched pathways are complement and coagulation cascades, drug metabolism and $A B C$ transporters (Table 3). For those genes over expressed in cell lines compared to tumors, the top three enriched cellular components are intermediate filament, intermediate filament cytoskeleton, and extracellular region, the top three enriched biological processes are G-protein coupled receptor protein signaling pathway, cell surface receptor linked signal transduction and sensory perception of smell, and the top one enriched pathway is Olfactory transduction (Table 4).

We find that the differentially expressed genes are primarily located in the extracellular space. Moreover, the genes related to immune systems are over expressed in tumors; an observation was previously reported [8]. Interestingly, many genes related to drug metabolism are also over expressed in HCC tumors as well. Liver is the primary organ for drug metabolism, whereas, the cell lines fail to express some of these genes. For example, CYP2C8, the primary enzyme for drug Paclitaxel, is expressed very low across all the HCC cell lines. Paclitaxel has very good sensitivity in vitro (with median IC50 $100 \mathrm{~nm}$, accessed in ChEMBL) but has no significant anti-tumor effect clinically [24]. The difference of its metabolism profiles between cell lines and tumors may shed light on different drug responses. The expression and activity of drug metabolism enzymes are demonstrated to be extremely low in HCC cell lines, particularly in HepG2 [25-28]. Although we see that the cell lines retain the expression of most genes, the differentially expressed genes between tumors and cell lines 


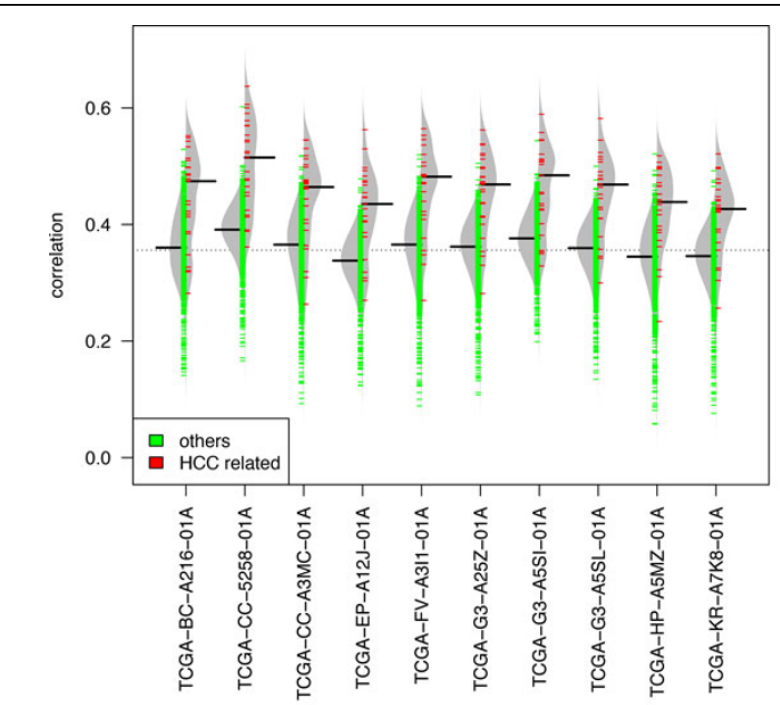

(a)

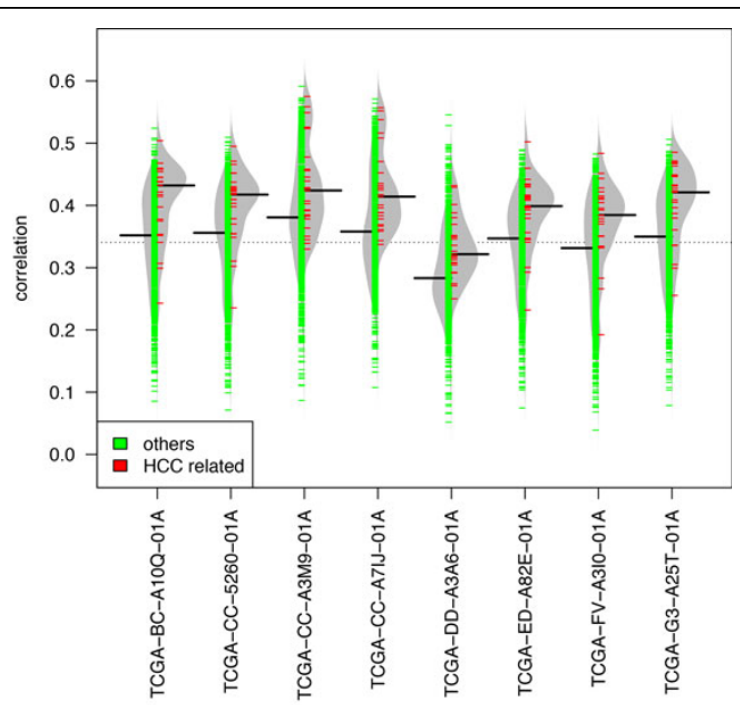

non-tumor:
tumor:

(b)

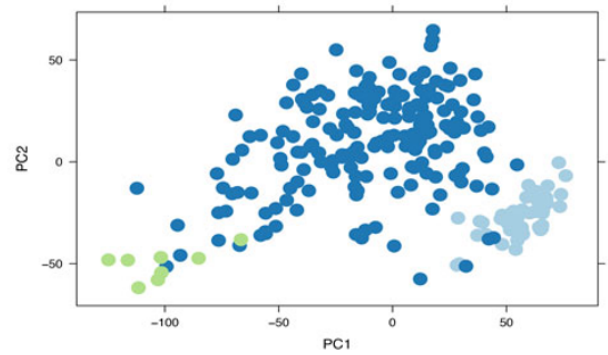

(c)

Figure 3 Example tumor samples significantly/poorly correlated to the HCC cell lines. (a) Ten tumor samples significantly correlated with the HCC cell lines. (b) Outlier tumor samples poorly correlated with the HCC cell lines. (c) Principal Component Analysis of the tumors (dark blue and green) and non-tumor samples (light blue) from TCGA, the outlier samples in (b) are shown in green.

are very important to investigate while we translate our discovery from in vitro to in vivo.

\section{Discussion}

An increasing number of HCC cell lines have been developed for the study of cancer biology and translational research [29], however, only a few cell lines are often chosen for in vitro or in vivo studies due to limited experimental capability. Choosing appropriate cell lines for experimental testing is thus of critical importance. Unfortunately, crucial information about cell lines (e.g., metastasis status, hepatitis virus status, drug treatment, mutation) is often missing or ambiguous in cell line databases and relevant publications, which further confounds

Table 2. Eight tumors poorly correlated to HCC cell lines

\begin{tabular}{ccccccccc}
\hline Sample ID & Gender & Race & Tumor status & Vital status & Grade & Residual & Metastasis stage & Pathologic tumor stage \\
\hline TCGA-BC-A10Q-01A & Female & White & With Tumor & Dead & NA & R1 & MX & NA \\
\hline TCGA-CC-5260-01A & Female & Asian & Tumor Free & Alive & G1 & RX & M0 & Stage IIIC \\
\hline TCGA-G3-A25T-01A & Female & White & Tumor Free & Alive & G2 & R0 & M0 & Stage IIIA \\
\hline TCGA-CC-A3M9-01A & Male & Asian & Tumor Free & Alive & G3 & R0 & M0 & Stage IIIA \\
\hline TCGA-FV-A310-01A & Female & White & Tumor Free & Alive & G2 & R0 & M0 & Stage II \\
\hline TCGA-DD-A3A6-01A & Female & White & Tumor Free & Dead & G2 & R0 & M0 & Stage II \\
\hline TCGA-CC-A7IJ-01A & NA & NA & NA & NA & NA & NA & NA & NA \\
\hline TCGA-ED-A82E-01A & Female & Asian & Tumor Free & Alive & G2 & R0 & M0 & Stage IIIA \\
\hline Fisher test (P value) & 0.01 & 0.69 & 0.68 & 0.76 & 0.16 & 0.45 & 1 & 0.16 \\
\hline
\end{tabular}

Two-tailed Fisher's exact test was used for associating clinical features to the tumors. 
Table 3. Enriched GO terms and KEGG pathways for over expressed genes in tumors

\begin{tabular}{|c|c|c|c|}
\hline & Term & P Value & Benjamini $(<0.1)$ \\
\hline \multirow[t]{12}{*}{ Cellular Component } & GO:0005576 extracellular region & $8.85 \mathrm{E}-11$ & $2.22 \mathrm{E}-08$ \\
\hline & GO:0005615 extracellular space & $2.87 \mathrm{E}-08$ & $3.60 \mathrm{E}-06$ \\
\hline & GO:0044421 extracellular region part & 5.49E-08 & $4.59 \mathrm{E}-06$ \\
\hline & GO:0031226 intrinsic to plasma membrane & $3.24 \mathrm{E}-05$ & 2.03E-03 \\
\hline & GO:0005887 integral to plasma membrane & $4.03 \mathrm{E}-05$ & $2.02 \mathrm{E}-03$ \\
\hline & GO:0005886 plasma membrane & $5.35 \mathrm{E}-04$ & $2.21 \mathrm{E}-02$ \\
\hline & GO:0005626 insoluble fraction & $6.52 \mathrm{E}-04$ & $2.31 \mathrm{E}-02$ \\
\hline & GO:0005624 membrane fraction & $7.56 \mathrm{E}-04$ & $2.35 \mathrm{E}-02$ \\
\hline & GO:0044459 plasma membrane part & $1.44 \mathrm{E}-03$ & $3.94 \mathrm{E}-02$ \\
\hline & GO:0000267 cell fraction & $1.62 \mathrm{E}-03$ & $3.98 \mathrm{E}-02$ \\
\hline & GO:0005792 microsome & $2.90 \mathrm{E}-03$ & $6.42 \mathrm{E}-02$ \\
\hline & GO:0042598 vesicular fraction & $3.65 \mathrm{E}-03$ & 7.36E-02 \\
\hline \multirow[t]{15}{*}{ Biological Process } & GO:0006955 immune response & $4.00 \mathrm{E}-11$ & $6.31 \mathrm{E}-08$ \\
\hline & GO:0009611 response to wounding & $9.12 \mathrm{E}-10$ & 7.19E-07 \\
\hline & GO:0006952 defense response & $3.32 \mathrm{E}-09$ & $1.75 \mathrm{E}-06$ \\
\hline & GO:0002253 activation of immune response & $2.74 \mathrm{E}-07$ & $1.08 \mathrm{E}-04$ \\
\hline & GO:0006954 inflammatory response & 4.09E-07 & $1.29 \mathrm{E}-04$ \\
\hline & GO:0050778 positive regulation of immune response & 9.19E-07 & $2.42 \mathrm{E}-04$ \\
\hline & GO:0006956 complement activation & $1.26 \mathrm{E}-06$ & $2.83 \mathrm{E}-04$ \\
\hline & GO:0002541 activation of plasma proteins involved in acute inflammatory response & $1.52 \mathrm{E}-06$ & 2.99E-04 \\
\hline & GO:0006959 humoral immune response & $2.98 \mathrm{E}-06$ & $5.22 \mathrm{E}-04$ \\
\hline & GO:0006958 complement activation, classical pathway & $1.70 \mathrm{E}-05$ & $2.68 \mathrm{E}-03$ \\
\hline & GO:0002684 positive regulation of immune system process & 1.77E-05 & $2.54 \mathrm{E}-03$ \\
\hline & GO:0002526 acute inflammatory response & 2.09E-05 & $2.75 \mathrm{E}-03$ \\
\hline & GO:0002455 humoral immune response mediated by circulating immunoglobulin & $2.55 \mathrm{E}-05$ & $3.09 \mathrm{E}-03$ \\
\hline & GO:0048584 positive regulation of response to stimulus & $6.24 \mathrm{E}-05$ & $7.00 \mathrm{E}-03$ \\
\hline & GO:0045087 innate immune response & $8.20 \mathrm{E}-05$ & $8.58 \mathrm{E}-03$ \\
\hline \multirow[t]{3}{*}{ KEGG pathway } & hsa04610:Complement and coagulation cascades & 1.40E-07 & $1.66 \mathrm{E}-05$ \\
\hline & hsa00982:Drug metabolism & $1.78 \mathrm{E}-03$ & 9.99E-02 \\
\hline & hsa02010:ABC transporters & $2.12 \mathrm{E}-03$ & $8.02 \mathrm{E}-02$ \\
\hline
\end{tabular}

Table 4. Enriched GO terms and KEGG pathways for over expressed genes in cell lines

\begin{tabular}{|c|c|c|c|}
\hline & Term & P Value & Benjamini $(<0.1)$ \\
\hline \multirow[t]{4}{*}{ Cellular Component } & GO:0005882 intermediate filament & 4.05E-09 & $6.65 \mathrm{E}-07$ \\
\hline & GO:0045111 intermediate filament cytoskeleton & $5.54 \mathrm{E}-09$ & 4.54E-07 \\
\hline & GO:0005576 extracellular region & $2.00 \mathrm{E}-08$ & $1.10 \mathrm{E}-06$ \\
\hline & GO:0045095 keratin filament & $3.11 \mathrm{E}-06$ & 1.27E-04 \\
\hline \multirow[t]{8}{*}{ Biological Process } & GO:0007186 G-protein coupled receptor protein signaling pathway & $7.66 \mathrm{E}-11$ & $8.79 \mathrm{E}-08$ \\
\hline & GO:0007166 cell surface receptor linked signal transduction & $1.82 \mathrm{E}-07$ & $1.04 \mathrm{E}-04$ \\
\hline & GO:0007608 sensory perception of smell & $4.78 \mathrm{E}-07$ & $1.83 \mathrm{E}-04$ \\
\hline & GO:0007606 sensory perception of chemical stimulus & 5.94E-07 & $1.70 \mathrm{E}-04$ \\
\hline & GO:0050877 neurological system process & 7.86E-07 & $1.80 \mathrm{E}-04$ \\
\hline & GO:0007600 sensory perception & 9.35E-07 & $1.79 \mathrm{E}-04$ \\
\hline & GO:0050890 cognition & 8.63E-06 & $1.41 \mathrm{E}-03$ \\
\hline & GO:0042742 defense response to bacterium & $5.24 \mathrm{E}-04$ & $7.24 \mathrm{E}-02$ \\
\hline KEGG pathway & hsa04740:Olfactory transduction & 4.46E-09 & 2.14E-07 \\
\hline
\end{tabular}


the selection process. As the genomic and transcriptomic profiles of cancer cell lines and tumors become more publically available, using gene expression to relate cancer cell lines to tumor samples becomes a more useful tool to inform cell line selection and has been applied to other cancers (e.g., ovarian cancer [16]). Although gene expression is not the only factor, it can be used to exclude certain cell lines that do not share similar profiles with the tumors being studied.

Our gene expression analysis of tumors and cell lines demonstrates that HCC cell lines are very tissue specific. They resemble the majority of HCC tumors using top 5000 varying genes. This conclusion still holds if top 1000 varying genes are used (data not shown). The four widely used cell lines (i.e., HepG2, HuH 7, Hep3B and PLC/PRF/5) are highly correlated to the tumors. However, nearly half of the cell lines are strikingly not significantly correlated to the tumors. Poor differentiation or genomic damage by chemotherapy drugs may account for the poor correlations of some cell lines.

Our analysis also shows that there is substantial variation of individual tumor samples in regards to their correlations with cell lines. Intrinsic heterogeneity among samples may account for the variation. Specifically, we identify 8 out of 200 tumor samples as outliers that have poor correlations with the known HCC cell lines. It may suggest that the drug tested in these cell lines will most likely have a different clinical effect in the outlier patients. It is interesting to further associate these tumors to clinical features so that this subset of tumors can be easily identified clinically and personalized treatments can be designed. However, the current 8 samples are insufficient to draw a robust conclusion. It may be also interesting to associate them to other genetic features.

Even though many HCC cell lines have overall high correlations with HCC tumors, a large number of genes are actually absent or lowly expressed in cell lines. A significant number of these genes are involved in disease development. Some genes are related to immune system and pharmacokinetics including drug metabolism and drug transportation. It's known that immune system and pharmacokinetics are related to drug response [30]. Inducing missing or low expressed genes in cell lines by external agents may be a way to improve cell line models $[26,31]$.

In summary, by leveraging publicly available transcriptomic data, we relate individual HCC tumors and cancer cell lines. We demonstrate that the most commonly used HCC cell lines closely resemble HCC tumors based on their gene expression, and we also identify nearly half of HCC cell lines do not resemble HCC tumors. Even in the highly correlated cell lines, a significant number of genes that are critical for disease development or drug response are absent or expressed at low levels, therefore, additional attention should be paid to these genes in translational research. Our future work includes covering more tumor samples from pubic sources (e.g., Gene Expression Omnibus) and more cell lines (the Sanger Cancer Cell Line project [32]), analyzing genetic features (e.g., mutation, copy number variation, single nucleotide polymorphism), and linking the subtype of patients classified by clinical features or molecular features to cancer cell lines. Finally, this work serves as a model analysis that can be extended to other tumor types of interest.

\section{Methods}

\section{Datasets}

We downloaded the cell line gene expression file (CCLE_Expression_Entrez_2012-09-29.gct) and the annotation file (CCLE_sample_info_file_2012-10-18.txt) from the CCLE website (http://www.broadinstitute.org/ ccle). Gene symbols in the expression file were converted into GeneIDs using AILUN [33]. In total, we analyzed 1019 cell lines with gene expression and annotation data. We collected relevant data of the cell lines including age, sex, race and other characteristics from ATCC (http://www.atcc.org), HSRRB (http://cellbank.nibio.go.jp/english/), and KCLB (http://cellbank. snu.ac.kr).

The level 3 released gene expressions for RNASeqV2 of HCC were downloaded from GDAC (http://gdac. broadinstitute.org). The RSEM abundance estimation processed by the TCGA workgroup was used in the following analysis. The sample clinical data was downloaded from TCGA (https://tcga-data.nci.nih.gov/tcga/). In total, we analyzed HCC 200 tumor samples and 50 non-tumor samples. According to TCGA, tumors samples are the primary tumors, namely the tumors at the initial site of cancer. Normal tissue samples are matched to the anatomic site of the tumor but usually not matched to the participant. Without any notes, tumor samples are specifically referred to HCC. We also downloaded all the samples in expO (GSE2109) from Gene Expression Omnibus.

\section{Correlation between tumor samples and cell lines}

The top 5000 genes ranked by interquartile range across all cell lines were chosen. Among them, 136 genes that are not matched to any genes in tumor samples were ignored in the following analysis. Since the expressions of tumor samples and cell lines were derived from two different technologies and were transformed via different methods [34], we used the ranked-based spearman correlation to assess the similarity between cell lines and tumor samples. For each cell line, the median of its correlations with all tumors was computed. The medians of all cancer cell lines were normally distributed. According 
to the distribution of the correlations, the significance of the correlation of each cell line with tumor samples was computed (P value $<0.05$ ).

For each tumor sample, Mann-Whitney test was used to test the difference of its correlations with HCC cell lines and with all other cell lines. Random 1000 tissue samples from different origins were taken from expO and further used to correct the $\mathrm{P}$ value from the MannWhitney test. Only the tumors with $\mathrm{P}$ value better than $95 \%$ of the random tissue samples were considered as being significantly correlated to the HCC cell lines.

\section{Differentially expressed genes between tumors and cell lines}

The common genes between tumor samples and cell lines samples were ranked based on their expression in individual samples. T-test was used to assess the difference of the ranks between tumor samples and cell lines for individual genes and $\mathrm{P}$ values were adjusted by the Benjamini-Hochberg for multiple hypothesis testing. Fold change was computed as the ratio between the mean of ranks across tumor samples and the mean of ranks across cell lines. Log2 fold change $>2$ and q value $<=0.01$ were used to select differentially expressed genes.

\section{Differentially expressed genes between tumors and non- tumors}

DESeq [35] was used to normalize RNA-Seq data and compute differentially expressed genes. $\log 2$ fold change $>2$ and $\mathrm{q}$ value $<=0.01$ were used to select differentially expressed genes.

\section{Software tools}

All the analysis was conducted in $\mathrm{R}$ programming language. Bioconductor packages Beanplot, ggplot2 and pheatmap were used for visualization. GO and KEGG pathway enrichment analysis was conducted in DAVID [23] (Benjamini < 0.1).

\section{Additional material}

Additional file 1: Lists of differentially expressed genes. Table S1: genes up regulated in HCC tumors comparing to HCC cell lines. Table S2: genes down regulated in HCC tumors compared to HCC cell lines. Table S3: genes differentially expressed in tumors compared to nontumors. Table S4: genes up regulated in tumors compared to cell lines and dysregulated in tumors compared to non-tumors. Table S5: genes down regulated in tumors compared to cell lines and dysregulated in tumors compared to non-tumors.

\section{Competing interests}

The authors declare that they have no competing interests.

\section{Authors' contributions}

$B C, M S$ and $A J B$ conceived the study. BC performed the analysis and wrote the manuscript. BC, MS, HFM, DH and AJB discussed the results. All authors contributed to writing, reviewing, editing and approving the manuscript.

\section{Acknowledgements}

Publication of this article has been funded in part by the Lucile Packard Foundation for Children's Health, the Stanford Child Health Research Institute, the Hewlett Packard Foundation, and the National Institute of General Medical Sciences (R01 GM079719).

This article has been published as part of BMC Medical Genomics Volume 8 Supplement 2, 2015: Selected articles from the 4th Translational Bioinformatics Conference and the 8th International Conference on Systems Biology (TBC/ISB 2014). The full contents of the supplement are available online at http://www.biomedcentral.com/bmcmedgenomics/ supplements/8/S2

Published: 29 May 2015

\section{References}

1. Wilding $J$ L, Bodmer WF: Cancer cell lines for drug discovery and development. Cancer research 2014, 74(9):2377-2384.

2. Gazdar AF, Girard L, Lockwood WW, Lam WL, Minna JD: Lung cancer cell lines as tools for biomedical discovery and research. Journal of the National Cancer Institute 2010, 102(17):1310-1321.

3. Gillet JP, Varma S, Gottesman MM: The clinical relevance of cancer cell lines. Journal of the National Cancer Institute 2013, 105(7):452-458.

4. Gazdar AF, Gao B, Minna JD: Lung cancer cell lines: Useless artifacts or invaluable tools for medical science? Lung cancer 2010, 68(3):309-318.

5. Sandberg R, Ernberg I: Assessment of tumor characteristic gene expression in cell lines using a tissue similarity index (TSI). Proceedings of the National Academy of Sciences of the United States of America 2005, 102(6):2052-2057.

6. Wang H, Huang S, Shou J, Su EW, Onyia JE, Liao B, Li S: Comparative analysis and integrative classification of $\mathrm{NCl60}$ cell lines and primary tumors using gene expression profiling data. BMC Genomics 2006, 7:166.

7. Ertel A, Verghese A, Byers SW, Ochs M, Tozeren A: Pathway-specific differences between tumor cell lines and normal and tumor tissue cells. Molecular cancer 2006, 5(1):55.

8. Stein WD, Litman T, Fojo T, Bates SE: A Serial Analysis of Gene Expression (SAGE) database analysis of chemosensitivity: comparing solid tumors with cell lines and comparing solid tumors from different tissue origins. Cancer research 2004, 64(8):2805-2816.

9. Jahchan NS, Dudley JT, Mazur PK, Flores N, Yang D, Palmerton A, Zmoos AF, Vaka D, Tran KQ, Zhou M, et al: A drug repositioning approach identifies tricyclic antidepressants as inhibitors of small cell lung cancer and other neuroendocrine tumors. Cancer discovery 2013, 3(12):1364-1377.

10. Sirota M, Dudley JT, Kim J, Chiang AP, Morgan AA, Sweet-Cordero A, Sage J, Butte AJ: Discovery and preclinical validation of drug indications using compendia of public gene expression data. Sci Trans/ Med 2011, 3(96):96ra77.

11. Dudley JT, Sirota M, Shenoy M, Pai RK, Roedder S, Chiang AP, Morgan AA, Sarwal MM, Pasricha PJ, Butte AJ: Computational repositioning of the anticonvulsant topiramate for inflammatory bowel disease. Science translational medicine 2011, 3(96):96ra76.

12. Zerbini LF, Bhasin MK, de Vasconcellos JF, Paccez JD, Gu X, Kung AL, Libermann TA: Computational repositioning and preclinical validation of pentamidine for renal cell cancer. Mol Cancer Ther 2014, 13(7):1929-1941.

13. Dudley JT, Chen R, Butte AJ: Matching cancer genomes to established cell lines for personalized oncology. Pacific Symposium on Biocomputing Pacific Symposium on Biocomputing 2011, 243-252.

14. Cancer Genome Atlas Research N, Weinstein JN, Collisson EA, Mills GB, Shaw KR, Ozenberger BA, Ellrott K, Shmulevich I, Sander C, Stuart JM: The Cancer Genome Atlas Pan-Cancer analysis project. Nat Genet 2013, 45(10):1113-1120.

15. Barretina J, Caponigro G, Stransky N, Venkatesan K, Margolin AA, Kim S, Wilson CJ, Lehar J, Kryukov GV, Sonkin D, et al: The Cancer Cell Line Encyclopedia enables predictive modelling of anticancer drug sensitivity. Nature 2012, 483(7391):603-607. 
16. Domcke S, Sinha R, Levine DA, Sander C, Schultz N: Evaluating cell lines as tumour models by comparison of genomic profiles. Nature communications 2013, 4:2126.

17. Rahib L, Smith BD, Aizenberg R, Rosenzweig AB, Fleshman JM, Matrisian $L M$ : Projecting cancer incidence and deaths to 2030: the unexpected burden of thyroid, liver, and pancreas cancers in the United States. Cancer research 2014, 74(11):2913-2921.

18. Villanueva A, Llovet JM: Targeted therapies for hepatocellular carcinoma. Gastroenterology 2011, 140(5):1410-1426.

19. Bagi CM, Andresen CJ: Models of hepatocellular carcinoma and biomarker strategy. Cancers 2010, 2(3):1441-1452.

20. Lee JH, Ku JL, Park YJ, Lee KU, Kim WH, Park JG: Establishment and characterization of four human hepatocellular carcinoma cell lines containing hepatitis B virus DNA. World journal of gastroenterology : WJG 1999, 5(4):289-295

21. Expression Project for Oncology. [http://www.ncbi.nlm.nih.gov/geo/query/ acc.cgi?acc=GSE2109].

22. Marin JJ, Martinez-Becerra P, Rosales R, Monte MJ: Chemotherapy in the treatment of primary liver tumours. Cancer Therapy 2008, 6(2).

23. Huang da W, Sherman BT, Lempicki RA: Systematic and integrative analysis of large gene lists using DAVID bioinformatics resources. Nat Protoc 2009, 4(1):44-57.

24. Chao Y, Chan WK, Birkhofer MJ, Hu OY, Wang SS, Huang YS, Liu M, WhangPeng J, Chi KH, Lui WY, et al: Phase II and pharmacokinetic study of paclitaxel therapy for unresectable hepatocellular carcinoma patients. $\mathrm{Br}$ J Cancer 1998, 78(1):34-39.

25. Wilkening S, Stahl F, Bader A: Comparison of primary human hepatocytes and hepatoma cell line Hepg2 with regard to their biotransformation properties. Drug Metab Dispos 2003, 31(8):1035-1042.

26. Westerink WM, Schoonen WG: Cytochrome P450 enzyme levels in HepG2 cells and cryopreserved primary human hepatocytes and their induction in HepG2 cells. Toxicology in vitro: an international journal published in association with BIBRA 2007, 21(8):1581-1591.

27. Brandon EF, Raap CD, Meijerman I, Beijnen JH, Schellens JH: An update on in vitro test methods in human hepatic drug biotransformation research: pros and cons. Toxicology and applied pharmacology 2003, 189(3):233-246.

28. Guo L, Dial S, Shi L, Branham W, Liu J, Fang JL, Green B, Deng H, Kaput J, Ning B: Similarities and differences in the expression of drugmetabolizing enzymes between human hepatic cell lines and primary human hepatocytes. Drug Metab Dispos 2011, 39(3):528-538.

29. Xin $H$, Wang $K$, Hu G, Xie F, Ouyang K, Tang X, Wang M, Wen D, Zhu Y, Qin X: Establishment and characterization of 7 novel hepatocellular carcinoma cell lines from patient-derived tumor xenografts. PloS one 2014, 9(1):e85308.

30. Igney FH, Krammer PH: Immune escape of tumors: apoptosis resistance and tumor counterattack. Journal of leukocyte biology 2002, 71(6):907-920

31. Mills JB, Rose KA, Sadagopan N, Sahi J, de Morais SM: Induction of drug metabolism enzymes and MDR1 using a novel human hepatocyte cell line. J Pharmacol Exp Ther 2004, 309(1):303-309.

32. Garnett MJ, Edelman EJ, Heidorn SJ, Greenman CD, Dastur A, Lau KW, Greninger P, Thompson IR, Luo X, Soares J, et al: Systematic identification of genomic markers of drug sensitivity in cancer cells. Nature 2012, 483(7391):570-575.

33. Chen R, Li L, Butte AJ: AlLUN: reannotating gene expression data automatically. Nat Methods 2007, 4(11):879.

34. Guo Y, Sheng Q, Li J, Ye F, Samuels DC, Shyr Y: Large scale comparison of gene expression levels by microarrays and RNAseq using TCGA data. PloS one 2013, 8(8):e71462.

35. Anders $\mathrm{S}$, Huber W: Differential expression analysis for sequence count data. Genome biology 2010, 11(10):R106.

doi:10.1186/1755-8794-8-S2-S5

Cite this article as: Chen et al: Relating hepatocellular carcinoma tumor samples and cell lines using gene expression data in translational research. BMC Medical Genomics 2015 8(Suppl 2):S5.

\section{Submit your next manuscript to BioMed Central and take full advantage of:}

- Convenient online submission

- Thorough peer review

- No space constraints or color figure charges

- Immediate publication on acceptance

- Inclusion in PubMed, CAS, Scopus and Google Scholar

- Research which is freely available for redistribution

Submit your manuscript at www.biomedcentral.com/submit
Biomed Central 